\title{
Quantitative analysis and clonal characterization of T-cell receptor $\beta$ repertoires in patients with advanced non-small cell lung cancer treated with cancer vaccine
}

\author{
TU MAI ${ }^{1,2^{*}}$, ATSUSHI TAKANO $^{3,4^{*}}$, HIROYUKI SUZUKI $^{5}$, TAKASHI HIROSE $^{6}$, TAKAHIRO MORI $^{7}$, \\ KOJI TERAMOTO ${ }^{3,4}$, KAZUMA KIYOTANI ${ }^{2}$, YUSUKE NAKAMURA ${ }^{1,2,8}$ and YATARO DAIGO Y $^{3,4}$ \\ ${ }^{1}$ Committee on Clinical Pharmacology and Pharmacogenomics; ${ }^{2}$ Section of Hematology and Oncology, \\ Department of Medicine, University of Chicago, Chicago, IL 60637, USA; ${ }^{3}$ Department of Medical Oncology; \\ ${ }^{4}$ Cancer Center, Shiga University of Medical Science, Otsu, Shiga 520-2192; ${ }^{5}$ Department of Regenerative Surgery,
} Fukushima Medical University, School of Medicine, Fukushima 960-1295; ${ }^{6}$ Division of Respiratory Medicine and Allergology, Department of Internal Medicine, Showa University School of Medicine, Shinagawa-ku, Tokyo 142-8555;

${ }^{7}$ Tohoku Community Cancer Services Program, Tohoku University Graduate School of Medicine, Sendai, Miyagi 980-8574, Japan; ${ }^{8}$ Department of Surgery, University of Chicago, Chicago, IL 60637, USA

Received June 18, 2016; Accepted March 3, 2017

DOI: $10.3892 / \mathrm{ol} .2017 .6125$

\begin{abstract}
With the development of cancer immunotherapy that may activate $\mathrm{T}$ cells, a practical and quantitative method to improve monitoring and/or prediction of immunological response of patients as a predictive biomarker is of importance. To examine possible biomarkers for a therapeutic cancer vaccine containing a mixture of three epitope peptides derived from cell division-associated 1 , lymphocyte antigen 6 complex locus $\mathrm{K}$ and insulin-like growth factor-II mRNA-binding protein 3, T-cell receptor $\beta$ (TCR $\beta$ ) repertoires of blood samples from 24 patients with human leukocyte antigen-A*2402-positive non-small cell lung cancer were characterized prior to and
\end{abstract}

Correspondence to: Professor Yusuke Nakamura, Section of Hematology and Oncology, Department of Medicine, University of Chicago, 5841 South Maryland Avenue, MC 2115, Chicago, IL 60637, USA

E-mail: ynakamura@bsd.uchicago.edu

Professor Yataro Daigo, Department of Medical Oncology and Cancer Center, Shiga University of Medical Science, Seta-Tsukinowa-Cho, Otsu, Shiga 520-2192, Japan

E-mail: ydaigo@belle.shiga-med.ac.jp

${ }^{*}$ Contributed equally

Abbreviations: NSCLC, non-small-cell lung cancer; TCR, T-cell receptor; HLA, human leukocyte antigen; CDR3, complementary-determining region 3; PBMC, peripheral blood mononuclear cell; PBL, peripheral blood lymphocyte; DI, diversity index

Key words: lung cancer, non-small cell lung cancer, cancer vaccine, T-cell receptor, next-generation sequencing, diversity index, immunogenicity following 8 weeks of the cancer vaccine treatment, by applying a next-generation sequencing method. It was identified that 14 patients with overall survival (OS) times of $\geq 12$ months had significantly lower TCR $\beta$ diversity indexes in samples prior to treatment, compared with 10 patients who succumbed within 1 year $(\mathrm{P}=0.03)$. In addition, patients with a high level of activated $\mathrm{CD}^{+} \mathrm{T}$ cells that are defined by a high granzyme $\mathrm{A} / \mathrm{CD} 8$ ratio had favorable $\mathrm{OS}$ rates (log-rank test, $\mathrm{P}=0.04$ ). The TCR $\beta$ diversity index and immunogenic gene markers following vaccine administration may serve as predictive or monitoring biomarkers for cancer vaccine treatment.

\section{Introduction}

Despite increasing knowledge about lung cancer and its treatment modalities over the last few decades, this disease continues to be responsible for the largest number of mortalities in males and females worldwide (1). Lung cancer was estimated to account for $27 \%$ of all cancer-associated mortalities in 2014 (2). There are two major histological types of lung cancer: Small cell lung cancer (SCLC) and non-SCLC (NSCLC) (3). Standard treatments for NSCLC include combinations of surgery, chemotherapy and radiotherapy (4). Although most advanced-stage patients receive chemotherapy and achieve clinical responses to a certain extent, the majority eventually experience relapse $(5,6)$. The 5-year survival rate of patients with NSCLC only marginally improved from 15.9 in 2008 to $18.0 \%$ in $2014(2,7,8)$. Therefore, novel therapies are required to ensure improved management of NSCLC.

Immunotherapy is a novel therapeutic strategy that is currently being evaluated for the treatment of NSCLC (4). Until recently, studies of vaccine treatment for patients with lung cancer have not yielded very positive results either due to non-specific immune system activation or due to toxicity (9-13). The lung, which has a high level of 
environmental exposure, has long been considered a poorly immunogenic tumor site (14). Therefore, an effective vaccine against NSCLC should contain antigens that are specific to the tumor cells and have the ability to generate immunogenicity following administration (15). Previously, cDNA microarray and laser microdissection were used to identify three genes for which transcripts were observed at high levels in cells from lung cancer, esophageal cancer, testis and placenta, but not in normal cells: Cell division-associated 1 (CDCA1) $(16,17)$, lymphocyte antigen 6 complex locus K (LY6K) $(17,18)$ and insulin-like growth factor-II mRNA-binding protein 3 (IMP-3) $(17,19)$ Accordingly, a vaccine comprising three human leukocyte antigen (HLA)-A24-restricted epitope peptides derived from these genes was developed for NSCLC and esophageal cancer (20). A Phase I clinical study of a combination of three peptides, including LY6K and IMP-3, in patients with advanced esophageal squamous cell carcinoma demonstrated that the vaccine was well tolerated and that strong T-cell responses to these specific antigens were induced following vaccination (21). Previously, a Phase II clinical study of a combination of three peptides (LY6K, IMP-3 and CDCA1) in patients with advanced head and neck squamous cell carcinoma also reported peptide-specific cytotoxic T lymphocyte (CTL) responses in the majority of the HLA-A2402-positive patients (22). In the present study, a Phase II clinical trial with exploratory investigations was conducted using a vaccine comprising these three peptides in patients with advanced NSCLC who were refractory to standard therapies (15).

One important predictive biomarker of vaccine therapy efficacy is the ability to induce an immunogenic response against specific cancer cells (23). Traditionally, enzyme-linked immune-spot and HLA-multimer assays have been used to measure CTL responses, since a high level of CTL infiltration into a tumor was reported to be associated with a good response to treatment (24). However, these assays require the ex vivo expansion of peripheral blood lymphocytes (PBLs) via stimulation and are not practical in clinical settings (25). In addition, lung tumor biopsy to examine intratumoral CTL infiltration is invasive (26). Therefore, it may be preferable to identify biomarkers from easily available human materials, including blood, using the predictive power that has been demonstrated to associate well with overall survival (OS) rate (27). The expansion and activation of certain T-cell populations, including cytotoxic $\mathrm{CD}^{+} \mathrm{T}$ cells, has been reported to be beneficial for the recognition and elimination of cancer cells $(28,29)$. By contrast, the expansion of regulatory $\mathrm{T}$ cells may be harmful, as these $\mathrm{T}$ cells protect cancer cells by suppressing tumor-specific $\mathrm{CD}^{+}$cytotoxic T cells (30-33). Therefore, it is important to quantitatively characterize the T-cell receptor (TCR) repertoires of patients with cancer prior to and following immunotherapy, including cancer vaccine treatment, to improve the understanding of the molecular mechanism underlying treatment efficacy.

In total $\sim 95 \%$ of $\mathrm{T}$ cells express TCR, which is a heterodimer of the TCR $\alpha$ and TCR $\beta$ chains, a signature of each T lymphocyte (34). To date, the TCR $\alpha$ gene on chromosome 14 has been reported to comprise a total of 70 variable (V) exons, 61 joining $(\mathrm{J})$ exons and 1 constant $(\mathrm{C})$ exon, whereas the TCR $\beta$ gene on chromosome 7 comprises $60 \mathrm{~V}$ exons, $15 \mathrm{~J}$ exons and $2 \mathrm{C}$ exons (35). In addition, TCR $\beta$ contains two diversity (D) exons; accordingly, the TCR genes undergo somatic $\mathrm{V}(\mathrm{D}) \mathrm{J}$ recombination, resulting in a significant increase in TCR diversity (36-38). This V(D)J segment rearrangement results in the highly variable complementary-determining region (CDR3), thus allowing the recognition of any possible antigens presented by HLA molecules (39). It has been estimated that $\sim 10^{18}$ different TCRs are generated in humans (25).

Advances in next-generation sequencing technology have made it possible to sequence millions of TCR cDNAs, and thus characterize patient TCR repertoires in a single experiment (40-42). In the present study, it was hypothesized that the expansion and activation of a large number of T-cell populations, particularly the cytotoxic T-cell population, may be used as predictive biomarkers in response to vaccine treatment to assess the outcomes of patients with NSCLC who had received cancer vaccine therapy. In the present study, T-cell repertoires and certain immune-associated molecules in patients with advanced NSCLC with an HLA-A*2402 who received the cancer vaccine treatment were characterized using cDNA-sequencing technology and a gene expression assay.

\section{Materials and methods}

Vaccines and patients. A total of 53 patients with advanced NSCLC resistant to standard therapies were enrolled between 21 May 2012 and 4 April 2013 in a Phase II open-label multicenter non-randomized clinical cancer vaccination trial conducted in an exploratory setting. Patients were vaccinated with a mixture of $1 \mathrm{mg}$ each of three HLA-A24-restricted epitope peptides derived from CDCA1, LY6K and IMP-3 mixed with incomplete Freund's adjuvant (Montanide ISA 51; SEPPIC, Puteaux, France) (trial no. NCT01592617). The clinical characteristics and treatment information for all 53 patients enrolled in the clinical trial are summarized in Table I. Patients received weekly subcutaneous injections of the peptides into the axillary region until disease progression was observed or the patient declined continued vaccine treatment. Written informed consent was obtained from all individuals enrolled in the trial. The trials were carried out in accordance with The Declaration of Helsinki on experimentation on human subjects, under the approval of the institutional ethics committees of the individual institutes. TCR $\beta$ sequencing and gene expression analysis were performed for blood samples from 24/35 HLA-A*2402-positive patients obtained.

Peripheral blood mononuclear cell collection. Blood samples were collected from patients prior to and following 8 weeks of vaccine treatment. Blood was drawn into BD Vacutainer ${ }^{\circledR}$ $\mathrm{CPT}^{\mathrm{TM}}$ cell preparation tubes containing sodium citrate (CPT; BD Biosciences, Franklin Lakes, NJ, USA). Samples were immediately centrifuged at $400 \mathrm{x} g$ for $20 \mathrm{~min}$ at room temperature. Peripheral blood mononuclear cells (PBMCs) were collected from the second layer of the column and washed with PBS. Cell numbers were determined using a hemocytometer, and cell viability was assessed via trypan blue staining. Following treatment with trypan blue, cells inside the four large corner squares were counted at x100 magnification under the light microscope (CKX41; Olympus Corporation, Tokyo, Japan). A total of $\sim 8 \times 10^{6}$ viable cells/sample were used for RNA isolation. 
RNA isolation and polymerase chain reaction (PCR) amplification. An RNeasy mini kit (Qiagen, Inc., Valencia, CA, USA) was used to isolate total RNA from PBMCs. In total $10 \mu 1$ DNase I (from the RNeasy mini kit) treatment was applied to remove undesirable genomic DNA contamination. A SMART cDNA library construction kit (Clontech Laboratories, Inc., Mountain View, CA, USA) was used to synthesize cDNA with a common adaptor (SMART IV oligonucleotide) at the 5'-ends. PCRs were performed to amplify TCR $\beta$ cDNAs. All possible TCR $\beta$ combinations were captured using a common forward primer (5'-GTCTCGTGGGCTCGGAGATGTGTATAAGAG ACAGTATCAACGCAGAGTGGCCAT-3') complementary to the SMART IV adaptor and a reverse primer specific for the constant region (5'-TCGTCGGCAGCGTCAGATGTGTAT AAGAGACAGDVHDVTCTGATGGCTCAAACACAGC-3'). The PCR protocol was as follows: $94^{\circ} \mathrm{C}$ for $3 \mathrm{~min}$; followed by 30 cycles of $94^{\circ} \mathrm{C}$ for $30 \mathrm{sec}, 65^{\circ} \mathrm{C}$ for $30 \mathrm{sec}$ and $72^{\circ} \mathrm{C}$ for $1 \mathrm{~min}$. Size selection (between 300 and 950 base pairs) was conducted using Pippin Prep (Sage Science, Beverly, MA, USA) to collect products known to be of the expected size (43). This experiment was performed once with the patient blood sample.

Library preparation and sequencing. A Nextera XT DNA library kit (Illumina, Inc., San Diego, CA, USA) was used to add adapter sequences onto template DNA to generate multiplexed sequencing libraries, allowing the sequencing and distinction of multiple samples in a single experiment. The PCR protocol was as follows: $95^{\circ} \mathrm{C}$ for $3 \mathrm{~min} ; 8$ cycles of $95^{\circ} \mathrm{C}$ for $30 \mathrm{sec}, 55^{\circ} \mathrm{C}$ for $30 \mathrm{sec}$ and $72^{\circ} \mathrm{C}$ for $30 \mathrm{sec}$; and a hold step at $72^{\circ} \mathrm{C}$ for $5 \mathrm{~min}$. Multiple dual index-encoded samples were combined in a single sequencing library. This library was loaded onto the MiSeq Reagent kit (version 3; Illumina, Inc.) and sequenced using an Illumina MiSeq Desktop Sequencer (Illumina, Inc.).

TCR sequence analysis. FASTQ files containing sequencing reads were generated using the MiSeq sequencer and mapped to the reference sequences derived from IMGT/GENE-DB (www.imgt.org) with the Bowtie2 alignment program (version 2.1.0; http://bowtie-bio.sourceforge.net/bowtie2/index . shtml) (44). To determine CDR3 in TCR $\beta$, a conserved cysteine residue encoded in the $3^{\prime}$ end of the $\mathrm{V}$ segment and a conserved phenylalanine residue encoded in the $5^{\prime}$ end of the J segment, which signal CDR3, were identified as described previously (25). Amino acid sequences were determined using the nucleotide sequences between the conserved TCR V cysteine residue and TCR J phenylalanine residue.

Gene expression assay. First-strand cDNA products as aforementioned were used in a gene expression assay to analyze the expression of CD4, CD8 and granzyme A (GZMA) transcripts. A PCR TaqMan gene expression assay was performed on an ABI ViiA ${ }^{\mathrm{TM}} 7$ system (Applied Biosystems; Thermo Fisher Scientific, Inc.), according to the manufacturer's protocol. All CD4, CD8 and GZMA transcript expression levels were normalized to the transcript expression of the housekeeping gene GAPDH.

Statistical analysis. OS rates were analyzed using the Kaplan-Meier estimator method, and survival was measured in days between the first vaccination and mortality. Progression-free survival (PFS) was measured in days from the first vaccination to the date of first documented disease progression or the date of mortality from any cause. The statistical significance of the survival period was analyzed using the Harrington-Fleming test.

The diversity indexes (DIs) of CDR3 sequences were calculated using inverse Simpson's index formula as previously reported (25). The DI reflects the total number and also the evenness of the identified clonotypes.

The Mann-Whitney test was used to compare the DIs between the long-survival and short-survival groups. A paired Student's $t$-test was performed to compare the DI prior to and following vaccine treatment within each group. These statistical tests were conducted using Prism software (version 6.0; GraphPad, Inc., La Jolla, CA, USA). The median was used as a threshold point to divide into two groups: High GZMA/CD8 and low GZMA/CD8 or high CD8/CD4 and low CD8/CD4. A log-rank test was performed using $\mathrm{R}$ software (version 3.2.0; The R Project for Statistical Computing, Vienna, Austria) to compare the percentage survival in these two groups. Data are presented as the mean \pm standard error. $\mathrm{P}<0.05$ was considered to indicate a statistically significant difference.

\section{Results}

Association between the TCR $\beta$ DI and survival status of patients with NSCLC treated with cancer peptide vaccines. Patients with NSCLC (35 HLA-A*2402-positive and 18 others) who had previously failed standard therapies were recruited, as summarized in Table I. The median OS time of these patients was 400 days, whereas the PFS was 57 days. No significant difference in the clinical outcomes between the HLA-A*2402-positive and -negative groups was identified. None of the patients exhibited a complete response according to the Response Evaluation Criteria in Solid Tumors (45). The most common adverse events of any grades observed during the present study are described in Table II.

Blood samples from 24 of 35 HLA-A*2402-positive patients were obtained, 14 of which achieved stable disease and/or remained alive $>12$ months after enrollment, and possible predictive immune biomarkers, including TCR $\beta$ analysis, were examined. The TCR $\beta$ DIs were compared between long-term survivors (lived for $>12$ months after enrollment) and short-term survivors (died within 12 months of enrollment) by calculating an inverse Simpson's DI (1/D) of the TCR $\beta$ repertoire, as described in the Materials and methods section. The present data revealed that prior to vaccine treatment, patients in the long-term survival group exhibited significantly lower TCR $\beta$ DIs compared with short-term survivors (63 \pm 61 vs. $259 \pm 307$; $\mathrm{P}=0.03$; Fig. $1 \mathrm{~A}$ ). However, the difference between the two groups was not significant in blood samples following 8 weeks of treatment $(107 \pm 108$ vs. $188 \pm 165 ; \mathrm{P}=0.12$; Fig. $1 \mathrm{~B})$ although a trend in the increase in DI was observed in the long-term survival group (mean of differences, 44 \pm ; P=0.07; Fig. 1C). By contrast, no significant change in DI between samples prior to and following the treatment was observed in the short-term survival group (Fig. 1D). The difference in the changes in DI in the long-term and short-term groups is presented in Fig. 1E. 
Table I. Background of patients.

\begin{tabular}{lccr}
\hline Characteristics & Total, $\mathrm{n}$ & HLA-A*2402(+), $\mathrm{n}$ & HLA-A*2402(-), $\mathrm{n}$ \\
\hline Total & 53 & 35 & 18 \\
Median age \pm SD, years & $64.0 \pm 7.7$ & $64.1 \pm 7.5$ & $63.9 \pm 8.2$
\end{tabular}

Sex

Female $\quad 21$

21

5

Male

32

19

13

Location of primary lesion

Pulmonary hilum

5

Lung field

43

Pleural effusion

Missing

1

4

5

0

28

15

0

2

1

Histological type

Adenocarcinoma

43

28

Squamous cell carcinoma

9

Pleomorphic carcinoma

$\mathrm{T}$ factor

0

TX

19

2

T0

3

T1a

T1b

T2a

$\mathrm{T} 2 \mathrm{~b}$

T3

T4

$\mathrm{N}$ factor

NX

N0

$\mathrm{N} 1$

$\mathrm{N} 2$

N3

$\mathrm{M}$ factor

MX

M0

M1a

M1b

M1a+1b

ECOG performance status score
0

1

2

Smoking status

Current or former smoker

Never smoked

EGFR mutation status

Positive

Not detected

Not reported

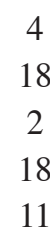

11

$$
0
$$

9

20

17

7

38

11

4

30

23

13

36

4
9

1

3

1

4

0

6

11

2

11

2

14

6

10

1

0

0

0

1

1

5

2

7

0

4

5

0

8

13

9

5

25

8

2

20

15

10

8

HLA, human leukocyte antigen; EGFR, epidermal growth factor receptor; T, tumor; N, node; M, metastasis; ECOG, Eastern Cooperative Oncology Group. 
Table II. Treatment-associated adverse effects.

\begin{tabular}{|c|c|c|c|c|c|c|}
\hline \multirow[b]{2}{*}{ Adverse events } & \multicolumn{2}{|c|}{ HLA-A*2402(+) $(n=35)$} & \multicolumn{2}{|c|}{ HLA-A $* 2402(-)(n=18)$} & \multicolumn{2}{|c|}{ Total $(n=53)$} \\
\hline & $\begin{array}{c}\text { Cases } \\
\text { (incidence rate), } \\
\mathrm{n}(\%)\end{array}$ & $\begin{array}{c}\text { Events, } \\
n\end{array}$ & $\begin{array}{c}\text { Cases } \\
\text { (incidence rate), } \\
\mathrm{n}(\%)\end{array}$ & $\begin{array}{c}\text { Events, } \\
\mathrm{n}\end{array}$ & $\begin{array}{c}\text { Cases } \\
\text { (incidence rate), } \\
\mathrm{n}(\%)\end{array}$ & $\begin{array}{c}\text { Events, } \\
\mathrm{n}\end{array}$ \\
\hline Total & $34(97.1)$ & 159 & $16(88.9)$ & 56 & $51(94.3)$ & 215 \\
\hline Infectious disease & $13(37.1)$ & 20 & $3(16.7)$ & 4 & $16(30.2)$ & 24 \\
\hline Nasopharyngitis & $6(17.1)$ & 7 & $1(5.6)$ & 1 & $7(13.2)$ & 8 \\
\hline Herpes zoster & $2(5.7)$ & 2 & $0(0.0)$ & 0 & $2(3.8)$ & 2 \\
\hline Periodontitis & $1(2.9)$ & 1 & $1(5.6)$ & 1 & $2(3.8)$ & 2 \\
\hline Bronchitis & $1(2.9)$ & 1 & $0(0.0)$ & 0 & $1(1.9)$ & 1 \\
\hline Cellulitis & $1(2.9)$ & 1 & $0(0.0)$ & 0 & $1(1.9)$ & 1 \\
\hline Influenza & $1(2.9)$ & 1 & $0(0.0)$ & 0 & $1(1.9)$ & 1 \\
\hline Esophageal candidiasis & $1(2.9)$ & 1 & $0(0.0)$ & 0 & $1(1.9)$ & 1 \\
\hline Paronychia & $0(0.0)$ & 0 & $1(5.6)$ & 1 & $1(1.9)$ & 1 \\
\hline Pneumonitis & $0(0.0)$ & 0 & $1(5.6)$ & 1 & $1(1.9)$ & 1 \\
\hline Pulpitis & $1(2.9)$ & 1 & $0(0.0)$ & 0 & $1(1.9)$ & 1 \\
\hline Rhinitis & $1(2.9)$ & 1 & $0(0.0)$ & 0 & $1(1.9)$ & 1 \\
\hline Foot tinea pedis & $1(2.9)$ & 1 & $0(0.0)$ & 0 & $1(1.9)$ & 1 \\
\hline Tonsillitis & $1(2.9)$ & 1 & $0(0.0)$ & 0 & $1(1.9)$ & 1 \\
\hline Urinary tract infection & $1(2.9)$ & 1 & $0(0.0)$ & 0 & $1(1.9)$ & 1 \\
\hline Device related infection & $1(2.9)$ & 1 & $0(0.0)$ & 0 & $1(1.9)$ & 1 \\
\hline Malignant neoplasm & $9(25.7)$ & 9 & $3(16.7)$ & 4 & $12(22.6)$ & 13 \\
\hline Malignant pleuritis & $2(5.7)$ & 2 & $2(11.1)$ & 2 & $4(7.5)$ & 4 \\
\hline Central nervous system metastasis & $2(5.7)$ & 2 & $0(0.0)$ & 0 & $2(3.8)$ & 2 \\
\hline NSCLC & $2(5.7)$ & 2 & $0(0.0)$ & 0 & $2(3.8)$ & 2 \\
\hline Malignant ascites & $1(2.9)$ & 1 & $0(0.0)$ & 0 & $1(1.9)$ & 1 \\
\hline Esophageal cancer & $1(2.9)$ & 1 & $0(0.0)$ & 0 & $1(1.9)$ & 1 \\
\hline Tumor infiltration of bone marrow & $1(2.9)$ & 1 & $0(0.0)$ & 0 & $1(1.9)$ & 1 \\
\hline Meningeal metastasis & $0(0.0)$ & 0 & $1(5.6)$ & 1 & $1(1.9)$ & 1 \\
\hline Brain tumor & $0(0.0)$ & 0 & $1(5.6)$ & 1 & $1(1.9)$ & 1 \\
\hline Metabolic disease & $2(5.7)$ & 2 & $0(0.0)$ & 0 & $2(3.8)$ & 2 \\
\hline Hyperuricemia & $1(2.9)$ & 1 & $0(0.0)$ & 0 & $1(1.9)$ & 1 \\
\hline Hyperlipidemia & $1(2.9)$ & 1 & $0(0.0)$ & 0 & $1(1.9)$ & 1 \\
\hline Mental disease & $1(2.9)$ & 1 & $1(5.6)$ & 1 & $2(3.8)$ & 2 \\
\hline Insomnia & $1(2.9)$ & 1 & $1(5.6)$ & 1 & $2(3.8)$ & 2 \\
\hline Nervous system disorder & $6(17.1)$ & 8 & $1(5.6)$ & 1 & $7(13.2)$ & 9 \\
\hline Headache & $3(8.6)$ & 3 & $0(0.0)$ & 0 & $3(5.7)$ & 3 \\
\hline Brain compression & $1(2.9)$ & 1 & $0(0.0)$ & 0 & $1(1.9)$ & 1 \\
\hline Convulsion & $1(2.9)$ & 2 & $0(0.0)$ & 0 & $1(1.9)$ & 2 \\
\hline Dizziness & $1(2.9)$ & 1 & $0(0.0)$ & 0 & $1(1.9)$ & 1 \\
\hline Hypoesthesia & $0(0.0)$ & 0 & $1(5.6)$ & 1 & $1(1.9)$ & 1 \\
\hline Drowsiness & $1(2.9)$ & 1 & $0(0.0)$ & 0 & $1(1.9)$ & 1 \\
\hline Eye disease & $1(2.9)$ & 1 & $1(5.6)$ & 1 & $2(3.8)$ & 2 \\
\hline Cataract & $1(2.9)$ & 1 & $0(0.0)$ & 0 & $1(1.9)$ & 1 \\
\hline Dry eye & $0(0.0)$ & 0 & $1(5.6)$ & 1 & $1(1.9)$ & 1 \\
\hline Ear disease and labyrinthine disturbance & $1(2.9)$ & 1 & $0(0.0)$ & 0 & $1(1.9)$ & 1 \\
\hline Rotary vertigo & $1(2.9)$ & 1 & $0(0.0)$ & 0 & $1(1.9)$ & 1 \\
\hline Heart disease & $2(5.7)$ & 4 & $0(0.0)$ & 0 & $2(3.8)$ & 4 \\
\hline Arrhythmia & $1(2.9)$ & 1 & $0(0.0)$ & 0 & $1(1.9)$ & 1 \\
\hline Pericardial effusion collection & $1(2.9)$ & 1 & $0(0.0)$ & 0 & $1(1.9)$ & 1 \\
\hline Angina & $1(2.9)$ & 1 & $0(0.0)$ & 0 & $1(1.9)$ & 1 \\
\hline
\end{tabular}


Table II. Continued.

\begin{tabular}{|c|c|c|c|c|c|c|}
\hline \multirow[b]{2}{*}{ Adverse events } & \multicolumn{2}{|c|}{ HLA-A*2402(+) $(n=35)$} & \multicolumn{2}{|c|}{ HLA-A $* 2402(-)(n=18)$} & \multicolumn{2}{|c|}{ Total $(n=53)$} \\
\hline & $\begin{array}{c}\text { Cases } \\
\text { (incidence rate), } \\
\mathrm{n}(\%)\end{array}$ & $\begin{array}{c}\text { Events, } \\
n\end{array}$ & $\begin{array}{c}\text { Cases } \\
\text { (incidence rate), } \\
\mathrm{n}(\%)\end{array}$ & $\begin{array}{c}\text { Events, } \\
\mathrm{n}\end{array}$ & $\begin{array}{c}\text { Cases } \\
\text { (incidence rate), } \\
\mathrm{n}(\%)\end{array}$ & $\begin{array}{c}\text { Events, } \\
n\end{array}$ \\
\hline Supraventricular tachycardia & $1(2.9)$ & 1 & $0(0.0)$ & 0 & $1(1.9)$ & 1 \\
\hline Angiopathy & $0(0.0)$ & 0 & $1(5.6)$ & 1 & $1(1.9)$ & 1 \\
\hline Hypertension & $0(0.0)$ & 0 & $1(5.6)$ & 1 & $1(1.9)$ & 1 \\
\hline Respiratory disease & $15(42.9)$ & 18 & $4(22.2)$ & 7 & $19(35.8)$ & 25 \\
\hline Upper respiratory infection & $11(31.4)$ & 13 & $2(11.1)$ & 4 & $13(24.5)$ & 17 \\
\hline Allergic rhinitis & $1(2.9)$ & 1 & $1(5.6)$ & 1 & $2(3.8)$ & 2 \\
\hline Atelectasis & $0(0.0)$ & 0 & $1(5.6)$ & 1 & $1(1.9)$ & 1 \\
\hline Voice disturbance & $1(2.9)$ & 1 & $0(0.0)$ & 0 & $1(1.9)$ & 1 \\
\hline Dyspnea & $0(0.0)$ & 0 & $1(5.6)$ & 1 & $1(1.9)$ & 1 \\
\hline Pleuritis & $1(2.9)$ & 1 & $0(0.0)$ & 0 & $1(1.9)$ & 1 \\
\hline Sneezing & $1(2.9)$ & 1 & $0(0.0)$ & 0 & $1(1.9)$ & 1 \\
\hline Hypertrophic rhinitis & $1(2.9)$ & 1 & $0(0.0)$ & 0 & $1(1.9)$ & 1 \\
\hline Gastrointestinal injury & $13(37.1)$ & 15 & $5(27.8)$ & 7 & $18(34.0)$ & 22 \\
\hline Constipation & $5(14.3)$ & 5 & $2(11.1)$ & 2 & $7(13.2)$ & 7 \\
\hline Diarrhea & $3(8.6)$ & 3 & $0(0.0)$ & 0 & $3(5.7)$ & 3 \\
\hline Vomiting & $1(2.9)$ & 1 & $2(11.1)$ & 3 & $3(5.7)$ & 4 \\
\hline Nausea & $2(5.7)$ & 2 & $0(0.0)$ & 0 & $2(3.8)$ & 2 \\
\hline Abdominal discomfort & $1(2.9)$ & 1 & $0(0.0)$ & 0 & $1(1.9)$ & 1 \\
\hline Enteritis & $0(0.0)$ & 0 & $1(5.6)$ & 1 & $1(1.9)$ & 1 \\
\hline Gastritis & $1(2.9)$ & 1 & $0(0.0)$ & 0 & $1(1.9)$ & 1 \\
\hline Hemorrhoid & $0(0.0)$ & 0 & $1(5.6)$ & 1 & $1(1.9)$ & 1 \\
\hline Esophageal stenosis & $1(2.9)$ & 1 & $0(0.0)$ & 0 & $1(1.9)$ & 1 \\
\hline Stomatitis & $1(2.9)$ & 1 & $0(0.0)$ & 0 & $1(1.9)$ & 1 \\
\hline Biliary system disorders & $1(2.9)$ & 1 & $0(0.0)$ & 0 & $1(1.9)$ & 1 \\
\hline Cholecystitis & $1(2.9)$ & 1 & $0(0.0)$ & 0 & $1(1.9)$ & 1 \\
\hline Skin disease & $7(20.0)$ & 9 & $1(5.6)$ & 1 & $8(15.1)$ & 10 \\
\hline Rash & $2(5.7)$ & 2 & $0(0.0)$ & 0 & $2(3.8)$ & 2 \\
\hline Alopecia & $0(0.0)$ & 0 & $1(5.6)$ & 1 & $1(1.9)$ & 1 \\
\hline Xerosis cutis & $1(2.9)$ & 1 & $0(0.0)$ & 0 & $1(1.9)$ & 1 \\
\hline Asteatotic eczema & $1(2.9)$ & 1 & $0(0.0)$ & 0 & $1(1.9)$ & 1 \\
\hline Erythema & $1(2.9)$ & 3 & $0(0.0)$ & 0 & $1(1.9)$ & 3 \\
\hline Skeletal muscle or soft tissue disorder & $5(14.3)$ & 5 & $1(5.6)$ & 2 & $6(11.3)$ & 7 \\
\hline Back pain & $1(2.9)$ & 1 & $0(0.0)$ & 0 & $1(1.9)$ & 1 \\
\hline Fasciitis & $0(0.0)$ & 0 & $1(5.6)$ & 2 & $1(1.9)$ & 2 \\
\hline Melalgia & $1(2.9)$ & 1 & $0(0.0)$ & 0 & $1(1.9)$ & 1 \\
\hline Periarthritis & $1(2.9)$ & 1 & $0(0.0)$ & 0 & $1(1.9)$ & 1 \\
\hline Spondylosis deformans & $1(2.9)$ & 1 & $0(0.0)$ & 0 & $1(1.9)$ & 1 \\
\hline Synovial cyst & $1(2.9)$ & 1 & $0(0.0)$ & 0 & $1(1.9)$ & 1 \\
\hline Kidney or urinary disorder & $0(0.0)$ & 0 & $1(5.6)$ & 1 & $1(1.9)$ & 1 \\
\hline Strangury & $0(0.0)$ & 0 & $1(5.6)$ & 1 & $1(1.9)$ & 1 \\
\hline Body or injection site disorder & $34(97.1)$ & 51 & $14(77.8)$ & 22 & $48(90.6)$ & 73 \\
\hline Injection site reaction & $33(94.3)$ & 41 & $13(72.2)$ & 20 & $46(86.8)$ & 61 \\
\hline High fever & $2(5.7)$ & 5 & $1(5.6)$ & 1 & $3(5.7)$ & 6 \\
\hline Disease progression & $2(5.7)$ & 2 & $1(5.6)$ & 1 & $3(5.7)$ & 3 \\
\hline General fatigue & $1(2.9)$ & 1 & $0(0.0)$ & 0 & $1(1.9)$ & 1 \\
\hline Injection site bleeding & $1(2.9)$ & 1 & $0(0.0)$ & 0 & $1(1.9)$ & 1 \\
\hline Pain & $1(2.9)$ & 1 & $0(0.0)$ & 0 & $1(1.9)$ & 1 \\
\hline
\end{tabular}


Table II. Continued.

\begin{tabular}{|c|c|c|c|c|c|c|}
\hline \multirow[b]{2}{*}{ Adverse events } & \multicolumn{2}{|c|}{ HLA-A $* 2402(+)(n=35)$} & \multicolumn{2}{|c|}{ HLA-A $* 2402(-)(n=18)$} & \multicolumn{2}{|c|}{ Total $(\mathrm{n}=53)$} \\
\hline & $\begin{array}{c}\text { Cases } \\
\text { (incidence rate), } \\
\mathrm{n}(\%)\end{array}$ & $\begin{array}{l}\text { Events, } \\
\mathrm{n}\end{array}$ & $\begin{array}{c}\text { Cases } \\
\text { (incidence rate), } \\
\mathrm{n}(\%)\end{array}$ & $\begin{array}{l}\text { Events, } \\
\mathrm{n}\end{array}$ & $\begin{array}{c}\text { Cases } \\
\text { (incidence rate), } \\
\mathrm{n}(\%)\end{array}$ & $\begin{array}{l}\text { Events, } \\
\mathrm{n}\end{array}$ \\
\hline Laboratory examination & $7(20.0)$ & 9 & $2(11.1)$ & 3 & $9(17.0)$ & 12 \\
\hline Elevated GTP $\gamma$ & $2(5.7)$ & 2 & $1(5.6)$ & 1 & $3(5.7)$ & 3 \\
\hline Lymphocytopenia & $2(5.7)$ & 2 & $0(0.0)$ & 0 & $2(3.8)$ & 2 \\
\hline Elevated hepatic transaminase & $0(0.0)$ & 0 & $1(5.6)$ & 1 & $1(1.9)$ & 1 \\
\hline Elevated CPK & $1(2.9)$ & 1 & $0(0.0)$ & 0 & $1(1.9)$ & 1 \\
\hline Elevated serum potassium level & $0(0.0)$ & 0 & $1(5.6)$ & 1 & $1(1.9)$ & 1 \\
\hline Elevated hepatic transaminase & $0(0.0)$ & 0 & $1(5.6)$ & 1 & $1(1.9)$ & 1 \\
\hline Increases in urine glucose levels & $1(2.9)$ & 1 & $0(0.0)$ & 0 & $1(1.9)$ & 1 \\
\hline Abnormal liver function test & $1(2.9)$ & 1 & $0(0.0)$ & 0 & $1(1.9)$ & 1 \\
\hline Albuminuria & $1(2.9)$ & 1 & $0(0.0)$ & 0 & $1(1.9)$ & 1 \\
\hline Weight loss & $1(2.9)$ & 1 & $0(0.0)$ & 0 & $1(1.9)$ & 1 \\
\hline $\begin{array}{l}\text { Injury, toxicosis and procedural } \\
\text { complication }\end{array}$ & $5(14.3)$ & 5 & $1(5.6)$ & 1 & $6(11.3)$ & 6 \\
\hline Fractured sacrum & $0(0.0)$ & 0 & $1(5.6)$ & 1 & $1(1.9)$ & 1 \\
\hline Lacerated wound & $1(2.9)$ & 1 & $0(0.0)$ & 0 & $1(1.9)$ & 1 \\
\hline Ligament sprain & $1(2.9)$ & 1 & $0(0.0)$ & 0 & $1(1.9)$ & 1 \\
\hline Ecchymoma & $1(2.9)$ & 1 & $0(0.0)$ & 0 & $1(1.9)$ & 1 \\
\hline Bruise & $1(2.9)$ & 1 & $0(0.0)$ & 0 & $1(1.9)$ & 1 \\
\hline Radiation dermatitis & $1(2.9)$ & 1 & $0(0.0)$ & 0 & $1(1.9)$ & 1 \\
\hline
\end{tabular}

HLA, human leukocyte antigen; CPK, creatine phosphokinase; NSCLC, non-small cell lung cancer.

A

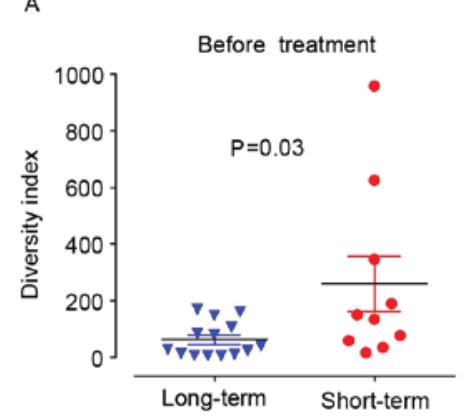

D

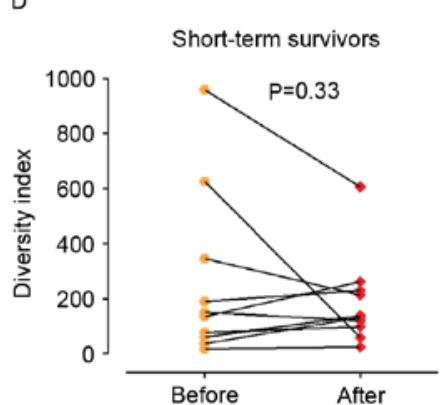

B

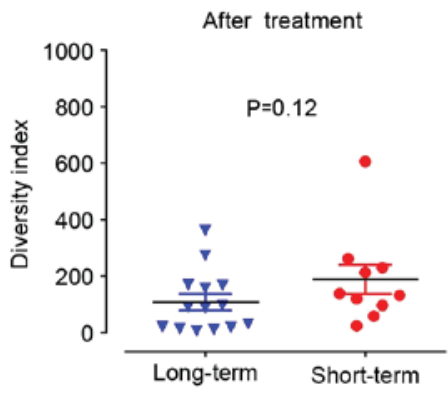

$E$

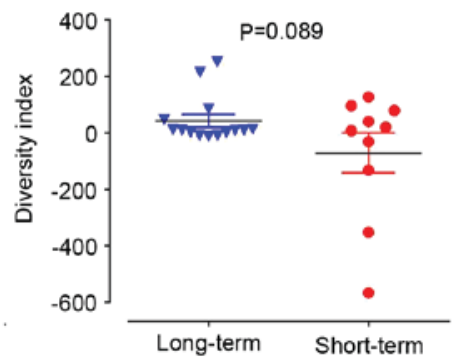

C

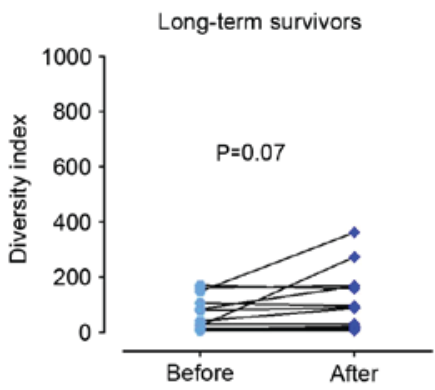

Figure 1. TCR $\beta$ diversity index analysis. (A) Diversity index prior to treatment. (B) Diversity index following treatment. Blue: Long-term survival group $(\mathrm{n}=14)$. Red: Short-term survival group $(\mathrm{n}=10)$. Data is presented as the mean \pm standard error. (C) Diversity indexes prior to and following treatment in the long-term survival group (alive $\geq 12$ months after treatment). (D) Diversity indexes prior to and following treatment in the short-term survival group (died within 12 months). (E) Changes of TCR $\beta$ diversity index following vaccine treatment. Blue: Long-term survival group ( $\mathrm{n}=14$ ). Red: Short-term survival group $(\mathrm{n}=10 ; \mathrm{P}=0.089)$. TCR $\beta$, T-cell receptor $\beta$. 
A

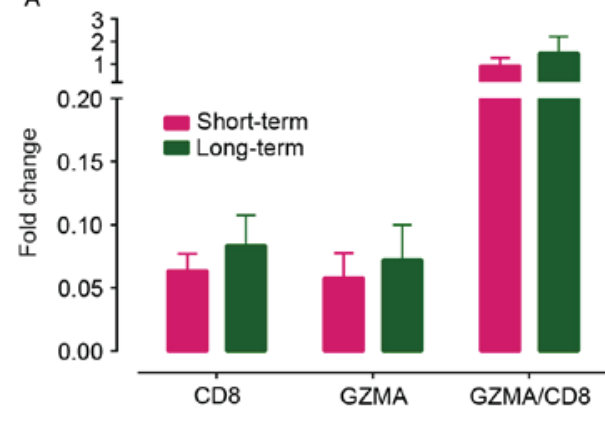

C

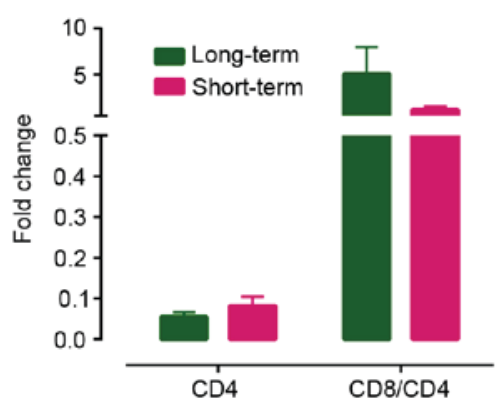

B

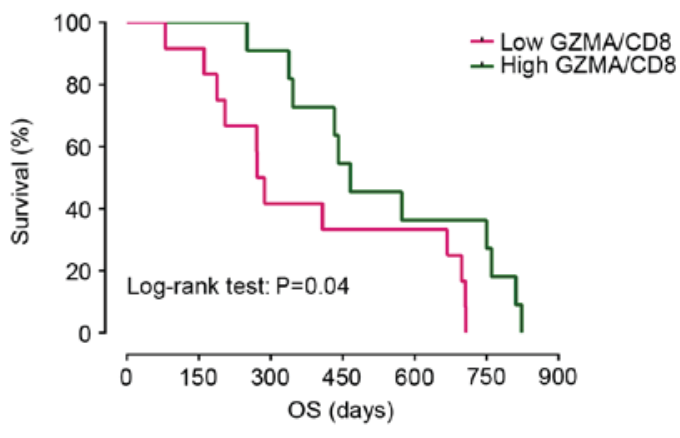

D

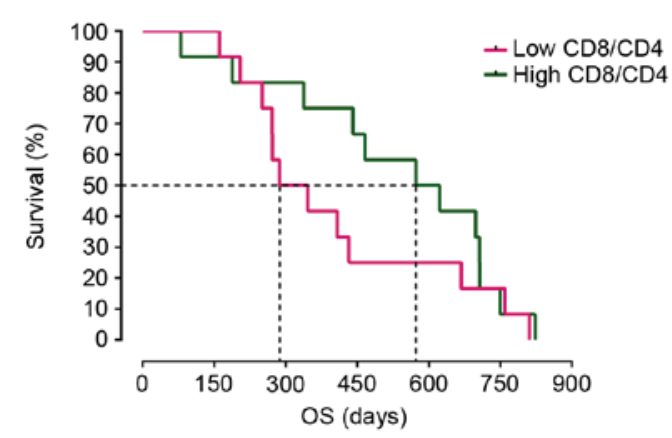

Figure 2. Gene expression analysis. (A) CD8 and GZMA expression normalized to GAPDH. Green: Long-term survival group (n=14). Pink: Short-term survival group ( $\mathrm{n}=10)$. (B) Kaplan-Meier estimator survival curve separated by the median into high and low GZMA/CD8 ratio prior to treatment (log-rank test, $\mathrm{P}=0.04)$. (C) $\mathrm{CD} 4$ expression levels and $\mathrm{CD} 8 / \mathrm{CD} 4$ ratio in the long- and short-term survival groups prior to treatment. Green: Long-term survival group ( $\mathrm{n}=14)$. Pink: Short-term survival group ( $\mathrm{n}=10$ ). (D) Kaplan-Meier survival curve separated by the median into high and low CD8/CD4 ratio prior to treatment. Median OS time: Low CD8/CD4 group, 316 days; high CD8/CD4 group, 598 days. GZMA, granzyme A; OS, overall survival; CD, cluster of differentiation.

Association between the immunogenic gene expression and survival status of patients with NSCLC treated with cancer peptide vaccines. To assess alterations in T-cell populations in response to treatment, TaqMan assays were performed to analyze the relative expression of CD8, CD4 and GZMA prior to the vaccine treatment. As presented in Fig. 2A, no significant differences were identified for CD8 and GZMA expression levels and the GZMA/CD8 ratio between the long-term and short-term survival groups. However, when the patients were separated into two groups by a median value of the GZMA/CD8 ratio, which may reflect the level of activated cytotoxic $\mathrm{T}$ cells, the survival curves were significantly different, favoring the group with a high GZMA/CD8 ratio (log-rank test, $\mathrm{P}=0.04$; Fig. 2B). In addition, a tendency was observed for the CD8/CD4 ratio to be increased in the long-term survival group compared with the short-term survival group (this difference was not statistically significant, Fig. 2C). Notably, the median overall survival time in the low CD8/CD4 group was only 316 days compared with 598 days in the high CD8/CD4 group (Fig. 2D).

\section{Discussion}

Although numerous attempts have been made to develop an effective peptide cancer vaccine therapy to treat NSCLC, the results have not been positive $(9,12,13)$. The present study aimed to screen possible predictive biomarkers that were able to distinguish responders from non-responders in patients with HLA-A24-positive NSCLC who received a cancer peptide vaccine treatment of a mixture of three peptides. Certain long-term survivors were observed among these patients who had previously failed standard therapies. Injection site reaction was the most common type of adverse event possibly associated with treatment. One patient in whom a coronary artery stent had been placed for angina succumbed to myocardial infarction as confirmed by autopsy, indicating a non-deniable causal association between treatment and this adverse event.

The immunogenic properties of this vaccine were evaluated through an analysis of the TCR $\beta$ repertoire in blood samples prior to and following 8 weeks of treatment. The main results included the following: i) A lower DI prior to vaccine treatment appeared to be beneficial, as it was associated with an improved survival status; and ii) a higher ratio of activated cytotoxic $\mathrm{T}$ cell at baseline, as indicated by GZMA/CD8 prior to treatment, appeared to be a favorable clinical outcome. A previous study demonstrated that cancer vaccination may cause an increase in circulating tumor antigen-specific $\mathrm{T}$ cells (46). However, the profiles of these $\mathrm{T}$ cells have not been well characterized with regard to their association with treatment outcomes. The results of the present study demonstrated that patients with a lower baseline DI tended to have a longer survival time. Notably, patients enrolled in the trial had advanced NSCLC refractory to standard chemotherapies. Prior treatment with cytotoxic chemotherapies may lead to generation of cancer-specific antigens that possibly generate immunological effects, although intensive chemotherapy may also kill these CTL clones (47). It was speculated that the residual immunogenic effects of prior chemotherapies led to the polyclonal expansion of T-cell clones and that this 
expansion, reflected as a lower DI of the TCR $\beta$ repertoire, contributed to the positive effect of the vaccine. Therefore, the pretreatment TCR $\beta$ DI may be used as a predictive marker of the ability of a patient to generate an immune response against either chemotherapeutic or vaccine treatment. Peptide vaccination was reported to boost a preexisting dominant clonotypic response (48). Therefore, a suggestion was made to administer vaccine therapy concomitantly with chemotherapy to generate the most effective immunological effects (49). A trend towards an increased TCR $\beta$ DI was observed in long-term survivors but not in short-term survivors, supporting earlier evidence that $\operatorname{TCR} \beta$ repertoire diversification due to chemotherapy and vaccination is beneficial in the prevention of immune-resistant mutant cancer cells, since more cancer-specific T-cell clones; particularly cytotoxic T cells are generated to target cancer cells (49). This phenomenon may be explained as a secondary immune response to vaccine treatment. As the immune system (either cytotoxic $\mathrm{T}$ cells or macrophages) eliminated greater numbers of cancer cells, phagocytosis of these cells may result in the presentation of cancer-specific antigens by antigen-presenting cells. The results of the present study were consistent with those of a study by Fang et al (25), in which patients with NSCLC were demonstrated to benefit from chemotherapy prior to peptide vaccination due to an increase in the TCR repertoire diversity. Therefore, the TCR $\beta$ DI prior to treatment and the increase in the DI of PBLs following treatment may be used to monitor the responses of patients with NSCLC to the peptide vaccine.

A TaqMan gene expression assay was performed to quantify the relative presence of these two populations in the patient samples in the present study. GZMA has been proposed as a biomarker of activated cytolytic $\mathrm{T}$ lymphocytes (50). A high level of GZMA expression indicates a high level of inflammatory cells in allograft, autoimmune diabetes and chronic Chagas' myocardial lesions $(51,52)$. Therefore, GZMA/CD8 ratios were calculated to study the activated subset of cytotoxic CD8 cells. Although no difference was observed in the expression of CD8 or GZMA between the long-term and short-term survivor groups, the results indicated that an increased level of GZMA/CD8 ratio was beneficial for the survival status. Although the difference was not statistically significant, the relative ratio of $\mathrm{CD}^{+} / \mathrm{CD}^{+}$was increased prior to treatment in the long-term survival group. A limitation of the present study was that the limited number of available T lymphocytes did not allow for T-cell sorting prior to sequencing. Therefore, future studies are required to improve the characterization of the specific TCR repertoires of different T-cell populations $\left(\mathrm{CD}^{+}\right.$, $\mathrm{CD} 4^{+} \mathrm{CD} 25^{+}$and $\mathrm{CD}^{+} \mathrm{CD} 25^{-}$), and thus elucidate the specific immunogenic effect of the three-peptide cocktail vaccine.

In conclusion, TCR $\beta$ DI and the immunogenic markers, including GZMA, may serve as predictive biomarkers for successful cancer vaccine treatment. Limitations of the present study included the limited number of patients and the lack of placebo control arm. Future prospective studies, in which these markers are used to predict the outcomes of vaccine treatment, are warranted.

\section{Acknowledgements}

The present study was partly supported by a research grant from the Japanese Ministry of Health Labor and Welfare.

\section{References}

1. Global status report on noncommunicable diseases 2014. World Health Organization, Geneva, pp11-14, 2014.

2. Cancer Facts \& Figures 2014. American Cancer Society, Atlanta, GA, pp14-15, 2014.

3. Travis WD, Brambilla E, Nicholson AG, Yatabe Y, Austin JH, Beasley MB, Chirieac LR, Dacic S, Duhig E, Flieder DB, et al: The 2015 World Health Organization Classification of Lung Tumors: Impact of Genetic, Clinical and Radiologic Advances Since the 2004 Classification. J Thorac Oncol 10: 1243-1260, 2015.

4. Santana-Davila R and Martins R: Treatment of stage IIIA non-small-cell lung cancer: A concise review for the practicing oncologist. J Oncol Pract 12: 601-606, 2016.

5. Katayama R, Shaw AT, Khan TM, Mino-Kenudson M, Solomon BJ, Halmos B, Jessop NA, Wain JC, Yeo AT, Benes C, et al: Mechanisms of acquired crizotinib resistance in ALK-rearranged lung Cancers. Sci Transl Med 4: 120ra17, 2012.

6. Kobayashi S, Boggon TJ, Dayaram T, Jänne PA, Kocher O, Meyerson M, Johnson BE, Eck MJ, Tenen DG and Halmos B: EGFR mutation and resistance of non-small-cell lung cancer to gefitinib. N Engl J Med 352: 786-792, 2005.

7. Ettinger DS, Akerley W, Borghaei H, Chang AC, Cheney RT, Chirieac LR, D'Amico TA, Demmy TL, Govindan R, Grannis FW Jr, et al: Non-small cell lung cancer, version 2.2013. J Natl Compr Canc Netw 11: 645-653, 2013.

8. Molina JR, Yang P, Cassivi SD, Schild SE and Adjei AA: Non-small cell lung cancer: Epidemiology, risk factors, treatment, and survivorship. Mayo Clin Proc 83: 584-594, 2008.

9. Butts C, Socinski MA, Mitchell PL, Thatcher N, Havel L, Krzakowski M, Nawrocki S, Ciuleanu TE, Bosquée L, Trigo JM, et al: Tecemotide (L-BLP25) versus placebo after chemoradiotherapy for stage III non-small-cell lung cancer (START): A randomized, double-blind, phase 3 trial. Lancet Oncol 15: 59-68, 2014.

10. Dessureault S, Noyes D, Lee D, Dunn M, Janssen W, Cantor A, Sotomayor E, Messina J and Antonia SJ: A phase-I trial using a universal GM-CSF-producing and CD40L-expressing bystander cell line (GM.CD40L) in the formulation of autologous tumor cell-based vaccines for cancer patients with stage IV disease. Ann Surg Oncol 14: 869-884, 2007.

11. Nemunaitis J, Nemunaitis M, Senzer N, Snitz P, Bedell C, Kumar P, Pappen B, Maples PB, Shawler D and Fakhrai H: Phase II trial of Belagenpumatucel-L, a TGF-beta 2 antisense gene modified allogeneic tumor vaccine in advanced non small cell lung cancer (NSCLC) patients. Cancer Gene Ther 16: 620-624, 2009.

12. Palmer M, Parker J, Modi S, Butts C, Smylie M, Meikle A, Kehoe M, MacLean G and Longenecker M: Phase I study of the BLP25 (MUC1 peptide) liposomal vaccine for active specific immunotherapy in stage IIIB/IV non-small-cell lung cancer. Clin Lung Cancer 3: 49-58, 2001.

13. Quoix E, Ramlau R, Westeel V, Papai Z, Madroszyk A, Riviere A, Koralewski P, Breton JL, Stoelben E, Braun D, et al: Therapeutic vaccination with TG4010 and first-line chemotherapy in advanced non-small-cell lung cancer: A controlled phase 2B trial. Lancet Oncol 12: 1125-1133, 2011

14. Zhang N, Zhong WZ and Wu YL: Special issue on personalized therapy in lung cancer. Transl Lung Cancer Res 3: 358-359, 2014.

15. Daigo Y, Takano A, Teramoto K, Chung S and Nakamura Y: A systematic approach to the development of novel therapeutics for lung cancer using genomic analyses. Clin Pharmacol Ther 94: 218-223, 2013.

16. Hayama S, Daigo $\mathrm{Y}$, Kato T, Ishikawa N, Yamabuki T, Miyamoto M, Ito T, Tsuchiya E, Kondo S and Nakamura Y: Activation of CDCA1-KNTC2, members of centromere protein complex, involved in pulmonary carcinogenesis. Cancer Res 66: 10339-10348, 2006.

17. Kikuchi T, Daigo Y, Katagiri T, Tsunoda T, Okada K, Kakiuchi S, Zembutsu H, Furukawa Y, Kawamura M, Kobayashi K, et al: Expression profiles of non-small cell lung cancers on cDNA microarrays: Identification of genes for prediction of lymph-node metastasis and sensitivity to anti-cancer drugs. Oncogene 22: 2192-2205, 2003.

18. Ishikawa N, Takano A, Yasui $\mathrm{W}$, Inai $\mathrm{K}$, Nishimura $\mathrm{H}$, Ito $\mathrm{H}$, Miyagi Y, Nakayama H, Fujita M, Hosokawa M, et al: Cancer-testis antigen lymphocyte antigen 6 complex locus $\mathrm{K}$ is a serologic biomarker and a therapeutic target for lung and esophageal carcinomas. Cancer Res 67: 11601-11611, 2007. 
19. Tomita Y, Harao M, Senju S, Imai K, Hirata S, Irie A, Inoue M Hayashida Y, Yoshimoto K, Shiraishi K, et al: Peptides derived from human insulin-like growth factor-II mRNA binding protein 3 can induce human leukocyte antigen-A2-restricted cytotoxic $\mathrm{T}$ lymphocytes reactive to cancer cells. Cancer Sci 102: 71-78, 2011.

20. Suda T, Tsunoda T, Daigo Y, Nakamura $Y$ and Tahara H: Identification of human leukocyte antigen-A24-restricted epitope peptides derived from gene products upregulated in lung and esophageal cancers as novel targets for immunotherapy. Cancer Sci 98: 1803-1808, 2007.

21. Kono K, Mizukami Y, Daigo Y, Takano A, Masuda K, Yoshida K, Tsunoda T, Kawaguchi Y, Nakamura Y and Fujii H: Vaccination with multiple peptides derived from novel cancer-testis antigens can induce specific T-cell responses and clinical responses in advanced esophageal cancer. Cancer Sci 100: 1502-1509, 2009.

22. Yoshitake Y, Fukuma D, Yuno A, Hirayama M, Nakayama H, Tanaka T, Nagata M, Takamune Y, Kawahara K, Nakagawa Y, et al: Phase II clinical trial of multiple peptide vaccination for advanced head and neck cancer patients revealed induction of immune responses and improved OS. Clin Cancer Res 21: 312-321, 2015.

23. Harrop R: Cancer vaccines: Identification of biomarkers predictive of clinical efficacy. Hum Vaccin Immunother 9: 800-804, 2013.

24. Grivennikov SI, Greten FR and Karin M: Immunity, inflammation, and cancer. Cell 140: 883-899, 2010.

25. Fang H, Yamaguchi R, Liu X, Daigo Y, Yew PY, Tanikawa C, Matsuda K, Imoto S, Miyano S and Nakamura Y: Quantitative $\mathrm{T}$ cell repertoire analysis by deep cDNA sequencing of $\mathrm{T}$ cell receptor $\alpha$ and $\beta$ chains using next-generation sequencing (NGS). Oncoimmunology 3: e968467, 2015.

26. Wong AK and Walkey AJ: Open lung biopsy among critically Ill, mechanically ventilated patients: A Metaanalysis. Ann Am Thorac Soc 12: 1226-1230, 2015.

27. Mehta S, Shelling A, Muthukaruppan A, Lasham A, Blenkiron C, Laking $G$ and Print C: Predictive and prognostic molecular markers for cancer medicine. Ther Adv Med Oncol 2: 125-148, 2010.

28. Zhang G, Zhao H, Wu J, Li J, Xiang Y, Wang G, Wu L and Jiao S: Adoptive immunotherapy for non-small cell lung cancer by NK and cytotoxic T lymphocytes mixed effector cells: Retrospective clinical observation. Int Immunopharmacology 21: 396-405, 2014.

29. Santegoets SJ, Turksma AW, Suhoski MM, Stam AG, Albelda SM, Hooijberg E, Scheper RJ, van den Eertwegh AJ, Gerritsen WR, Powell DJ Jr, et al: IL-21 promotes the expansion of $\mathrm{CD} 27^{+} \mathrm{CD} 28^{+}$tumor infiltrating lymphocytes with high cytotoxic potential and low collateral expansion of regulatory T cells. J Transl Med 11: 37, 2013

30. Khazaie $\mathrm{K}$ and von Boehmer $\mathrm{H}$ : The impact of $\mathrm{CD} 4^{+} \mathrm{CD} 25^{+}$Treg on tumor specific $\mathrm{CD} 8^{+} \mathrm{T}$ cell cytotoxicity and cancer. Semin Cancer Biol 16: 124-136, 2006.

31. Erfani N, Mehrabadi SM, Ghayumi MA, Haghshenas MR, Mojtahedi Z, Ghaderi A and Amani D: Increase of regulatory $\mathrm{T}$ cells in metastatic stage and CTLA-4 over expression in lymphocytes of patients with non-small cell lung cancer (NSCLC). Lung Cancer 77: 306-311, 2012.

32. Phillips JD, Knab LM, Blatner NR, Haghi L, DeCamp MM, Meyerson SL, Heiferman MJ, Heiferman JR, Gounari F, Bentrem DJ and Khazaie K: Preferential expansion of pro-inflammatory Tregs in human non-small cell lung cancer. Cancer Immunol Immunother 64: 1185-1191, 2015.

33. Gounaris E, Blatner NR, Dennis K, Magnusson F, Gurish MF, Strom TB, Beckhove P, Gounari F and Khazaie K: T-regulatory cells shift from a protective anti-inflammatory to a cancer-promoting proinflammatory phenotype in polyposis. Cancer Res 69: 5490-5497, 2009.

34. Glusman G, Rowen L, Lee I, Boysen C, Roach JC, Smit AF, Wang K, Koop BF and Hood L: Comparative genomics of the human and mouse $\mathrm{T}$ cell receptor loci. Immunity 15: 337-349, 2001 ,
35. Litman GW, Rast JP and Fugmann SD: The origins of vertebrate adaptive immunity. Nat Rev Immunol 10: 543-553, 2010.

36. Folch $\mathrm{G}$ and Lefranc MP: The human T cell receptor beta variable (TRBV) genes. Exp Clin Immunogenet 17: 42-54, 2000.

37. Haynes MR and Wu GE: Gene discovery at the human T-cell receptor alpha/delta locus. Immunogenetics 59: 109-121, 2007.

38. Scaviner D and Lefranc MP: The human T cell receptor alpha variable (TRAV) genes. Exp Clin Immunogenet 17: 83-96, 2000.

39. Robinson MW, Hughes J, Wilkie GS, Swann R, Barclay ST, Mills PR, Patel AH, Thomson EC and McLauchlan J: Tracking TCR $\beta$ sequence clonotype expansions during antiviral therapy using high-throughput sequencing of the hypervariable region. Front Immunol 7: 131, 2016.

40. Britanova OV, Putintseva EV, Shugay M, Merzlyak EM, Turchaninova MA, Staroverov DB, Bolotin DA, Lukyanov S, Bogdanova EA, Mamedov IZ, et al: Age-related decrease in TCR repertoire diversity measured with deep and normalized sequence profiling. J Immunol 192: 2689-2698, 2014.

41. Li Z, Liu G, Tong Y, Zhang M, Xu Y, Qin L, Wang Z, Chen X and He J: Comprehensive analysis of the T-cell receptor beta chain gene in rhesus monkey by high throughput sequencing. Sci Rep 5: 10092, 2015.

42. Li D, Gao G, Li Z, Sun W, Li X, Chen N, Sun J and Yang Y: Profiling the T-cell receptor repertoire of patient with pleural tuberculosis by high-throughput sequencing. Immunol Lett 162: 170-180, 2014.

43. Yew PY, Alachkar H, Yamaguchi R, Kiyotani K, Fang H, Yap KL, Liu HT, Wickrema A, Artz A, van Besien K, et al: Quantitative characterization of T-cell repertoire in allogeneic hematopoietic stem cell transplant recipients. Bone Marrow Transplant 50: 1227-1234, 2015.

44. Giudicelli V, Chaume D and Lefranc MP: IMGT/GENE-DB: A comprehensive database for human and mouse immunoglobulin and T cell receptor genes. Nucleic Acids Res 33 (Database Issue): D256-D261, 2005.

45. Eisenhauer EA, Therasse P, Bogaerts J, Schwartz LH, Sargent D, Ford R, Dancey J, Arbuck S, Gwyther S, Mooney M, et al: New response evaluation criteria in solid tumours: Revised RECIST guideline (version 1.1). Eur J Cancer 45: 228-247, 2009.

46. Spiotto MT and Schreiber H: Rapid destruction of the tumor microenvironment by CTLs recognizing cancer-specific antigens cross-presented by stromal cells. Cancer Immun 5: 8, 2005.

47. Bonmassar E, Bonmassar A, Vadlamudi S and Goldin A: Immunological alteration of leukemic cells in vivo after treatment with an antitumor drug. Proc Natl Acad Sci USA 66 1089-1095, 1970.

48. Speiser DE, Baumgaertner P, Barbey C, Rubio-Godoy V, Moulin A, Corthesy P, Devevre E, Dietrich PY, Rimoldi D, Liénard D, et al: A novel approach to characterize clonality and differentiation of human melanoma-specific T cell responses: Spontaneous priming and efficient boosting by vaccination. J Immunol 177: 1338-1348, 2006.

49. Palermo B, Del Bello D, Sottini A, Serana F, Ghidini C, Gualtieri N, Ferraresi V, Catricalà C, Belardelli F, Proietti E, et al: Dacarbazine treatment before peptide vaccination enlarges T-cell repertoire diversity of melan-a-specific, tumor-reactive CTL in melanoma patients. Cancer Res 70: 7084-7092, 2010.

50. Cornberg M, Chen AT, Wilkinson LA, Brehm MA, Kim SK, Calcagno C, Ghersi D, Puzone R, Celada F, Welsh RM and Selin LK: Narrowed TCR repertoire and viral escape as a consequence of heterologous immunity. J Clin Invest 116: 1443-1456, 2006.

51. Reis DD, Jones EM, Tostes S Jr, Lopes ER, Gazzinelli G, Colley DG and McCurley TL: Characterization of inflammatory infiltrates in chronic chagasic myocardial lesions: Presence of tumor necrosis factor-alpha ${ }^{+}$cells and dominance of granzyme $\mathrm{A}^{+}, \mathrm{CD}^{+}$lymphocytes. Am J Trop Med Hyg 48: 637-644, 1993.

52. Chen RH, Ivens KW, Alpert S, Billingham ME, Fathman CG, Flavin TF, Shizuru JA, Starnes VA, Weissman IL and Griffiths GM: The use of granzyme A as a marker of heart transplant rejection in cyclosporine or anti-CD4 monoclonal antibody-treated rats. Transplantation 55: 146-153, 1993. 